\title{
Protéases d'origine stromale et progression tumorale
}

\section{Catherine Wolf \\ Olivier Lefebvre \\ Nicolas Rouyer \\ Marie-Pierre Chenard \\ Jean-Pierre Bellocq \\ Marie-Christine Rio \\ Pierre Chambon \\ Paul Basset}

Les études les plus récentes, basées sur l'hybridation in situ d'ARNm, ont montré que dans de nombreux carcinomes humains les protéases extracellulaires étaient préférentiellement exprimées par les cellules stromales, et non par les cellules cancéreuses comme cela était généralement admis. Ces observations conduisent à reconsidérer le rôle joué par les protéases extracellulaires dans la progression tumorale, et renforcent l'idée selon laquelle le stroma des carcinomes représente une cible potentielle pour le développement de nouveaux agents anti-tumoraux.

\footnotetext{
ADRESSES

C. Wolf : docteur es sciences. O. Lefebvre : étu diant en doctorat. M-C. Rio: directeur de recherche à l'Inserm. P. Chambon : professeur des universités, praticien hospitalier. P. Basset professeur des universités, praticien hospilalier. Laboratoire de génétique moléculaire des eucaryotes du Cnrs, Unité 184 de biologie moléculaire et de génie génétique de l'Inserm, institut de chimie biologique, faculté de médecine, 11 , rue Humann, 67085 Strasbourg Cedex, France.

N. Rouyer : praticien hospitalier à titre provisoire, M-P. Chenard: maître de conférence, pra ticien hospitalier, J-P. Bellocq : professeur des universités, praticien hospitalier. Service d'anatomie pathologique générale, centre hospitalier universitaire, hôpital de Hautepierre, 1, avenue Molière, 67098 Strasbourg Cedex, France.

$\mathrm{m} / \mathrm{s} n^{\circ} 5$ vol. 10, mai 94
}

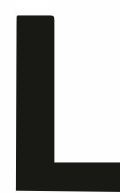

a grande majorité des tumeurs rencontrées en pathologie humaine sont des carcinomes, c'est-à-dire des tumeurs d'origine épithéliale qui évoluent généralement en plusieurs phases. Dans un premier temps, les cellules cancéreuses prolifèrent localement dans leur compartiment épithélial d'origine, séparées du reste de l'organisme par une membrane basale riche en collagène de type IV et en laminine [1]. Cette prolifération cancéreuse initiale, dite carcinome in situ, échappe souvent au diagnostic qui n'est posé que lorsque les cellules cancéreuses franchissent la membrane basale pour former une masse tumorale. On parle alors de carcinome invasif qui, dans bon nombre de cas, évoluera par dissémination métastatique, caractérisée par le développement de tumeurs secondaires à distance du site de la tumeur primaire [2].

Il est important de noter que dans les carcinomes humains les cellules cancéreuses invasives ne sont généralement pas au contact direct du tissu conjonctif normal, mais enchâssées dans un stroma tumoral se distinguant du tissu conjonctif normal tant sur le plan morphologique que biochimique [3, 4]. Il existe peu d'exceptions à ce schéma, les plus notables étant celles des carcinomes rénaux et des carcinomes pulmonaires à petites cellules, dont la progression s'effectue le plus souvent sans qu'il y ait de remaniement majeur du tissu conjonctif. La plupart des autres carcinomes humains présentent deux composantes, l'une épithéliale et l'autre stromale, participant toutes deux à la constitution de la masse tumorale.

\section{Protéolyse}

et progression tumorale

Le modèle classique d'invasion tumorale, tel qu'il a été popularisé par Liotta (Bethesda MD, USA) [5], fait intervenir trois étapes successives: une première étape d'attache- 


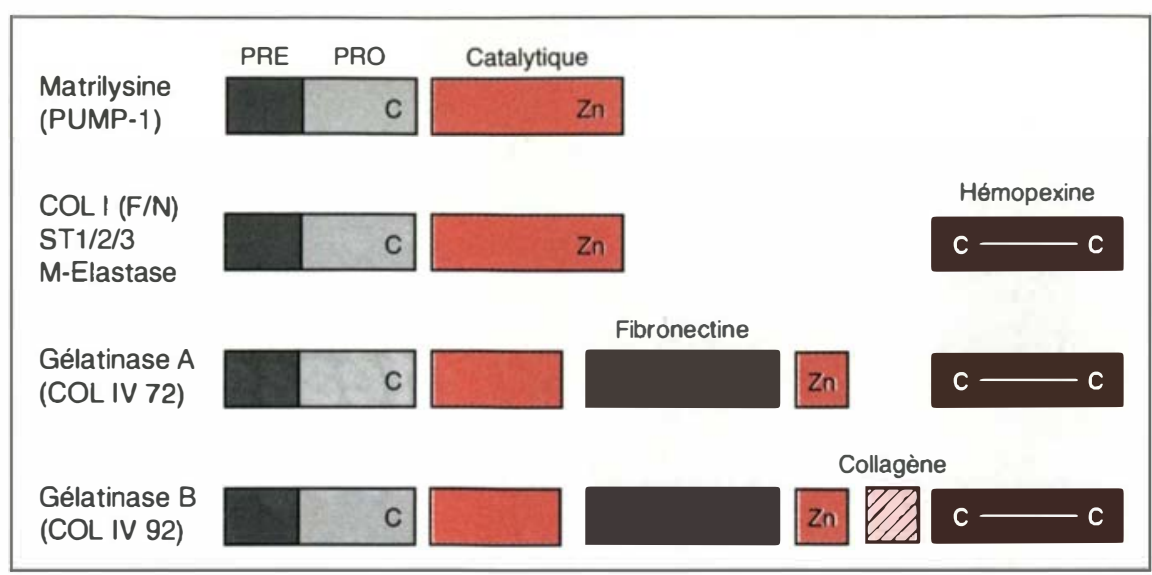

Figure 1. Domaines protéiques des métalloprotéases matricielles. La famille des métalloprotéases matricielles comprend actuellement neuf membres distincts chez l'homme [10]. Toutes les enzymes sont constituées d'au moins trois domaines protéiques qui sont, en partant de l'extrémité $\mathrm{NH}_{2}$-terminale, un peptide signal ou prédomaine (PRE), un prodomaine $(P R O)$ et un domaine catalytique. Ces enzymes, sauf la matrilysine (précédemment appelée PUMP-1), contiennent d'autres domaines protéiques qui jouent un rôle dans la spécificité vis-à-vis du substrat. Le domaine dit de I'hémopexine, len raison de son analogie de structure avec le transporteur de l'hème), intervient également dans l'interaction avec les TIMP (tissue inhibitor of metalloproteinase) et, dans le cas de la gélatinase $A$, ce domaine est requis pour l'activation de la pro-enzyme par les membranes cellulaires $[15,20]$. La latence des formes enzymatiques sécrétées est due à l'interaction entre l'atome de zinc du site catalytique et la cystéine du prodomaine, cystéine caractéristique des métalloprotéases matricielles au sein de l'ensemble des métalloprotéases. L'activation des zymogènes implique l'élimination du prodomaine, aboutissant à une forme active monomérique dans laquelle l'atome de zinc du site catalytique, libéré de sa liaison avec le résidu cystéine du prodomaine, peut interagir avec le substrat.

Abréviations: $C O L$ I (F/N): collagénase de type I $(F=$ fibroblastique $N=$ neutrophile); ST1/2/3: stromélysine-1/2/3; M-ELASTASE : métallo-élastase macrophagique; COL IV 72: collagénase de type IV de 72 kDa; COL IV 92: collagénase de type $N$ de $92 k D a ; C$ : cystéine; Hémopexine, Fibronectine, Collagène: domaines protéiques des métalloprotéases matricielles présen-

5. Liotta LA, Nageswara Rao C, Wewer UM. Biochemical interactions of tumor cells with the basement membrane. Annu Rou Biochem 1986; 55 : 1037-57.

6. Rochefort H, Capony F. Protéases lysosomiales et invasion tumorale. médecine/sciences $1991 ; 7: 30-6$.

7. Monsky WL, Chen WT. Proteases of cell adhesion proteins in cancer. Cancer Biol 1993; 4 : 251-8.

8. Mignatti P, Rifkin DB. Biology and biochemistry of proteinases in tumor invasion. Physiol Rer 1993; 73: 161-95.

9. Duffy MJ. Plasminogen activators and cancer. Blood Coagulation Fïrinolysis 1990 ; 1: 681-7.

10. Matrisian LM. The matrix-degrading metalloproteinases. Biolissays 1992; 14: 455-63.

11. Melchiori A, Albini A, Ray JM, StetlerStevenson WG. Inhibition of tumor cell invasion by a highly conserved peptide sequence from the matrix metalloproteinase enzyme prosegment. Cancer Res 1992

\section{2: $2353-6$}

\section{tant une homologie structurale avec ces protéines.}

ment, au cours de laquelle les cellules cancéreuses renforcent leur adhérence à la membrane basale épithéliale; une deuxième étape de protéolyse, caractérisée par la lyse de la membrane basale; une troisième étape, au cours de laquelle les cellules cancéreuses se mobilisent, lysent la matrice extracellulaire du compartiment stromal et envahissent l'espace tissulaire ainsi libéré. A ce stade d'invasion tumorale, les cellules cancéreuses peuvent disséminer par voie lymphatique ou sanguine, le franchissement des membranes basales endothéliales étant censé obéir à un schéma semblable à celui proposé pour le franchissement de la membrane basale délimitant le compartiment épithélial $[2,5]$.

Plusieurs types de protéases sont susceptibles d'être impliquées dans ces étapes successives de protéolyse. Il s'agit de protéases lysosomiales, dont le chef de file est la cathepsine D [6], et d'un certain nombre de protéases membranaires [7]. Toutefois, les enzymes protéolytiques les plus souvent associées à la notion de progression tumorale appartiennent à deux groupes de protéases sécrétées, les métalloprotéases matricielles et les enzymes du système de la plasmine. L'expression de ces protéases est en effet fréquemment augmentée dans les tissus cancéreux 


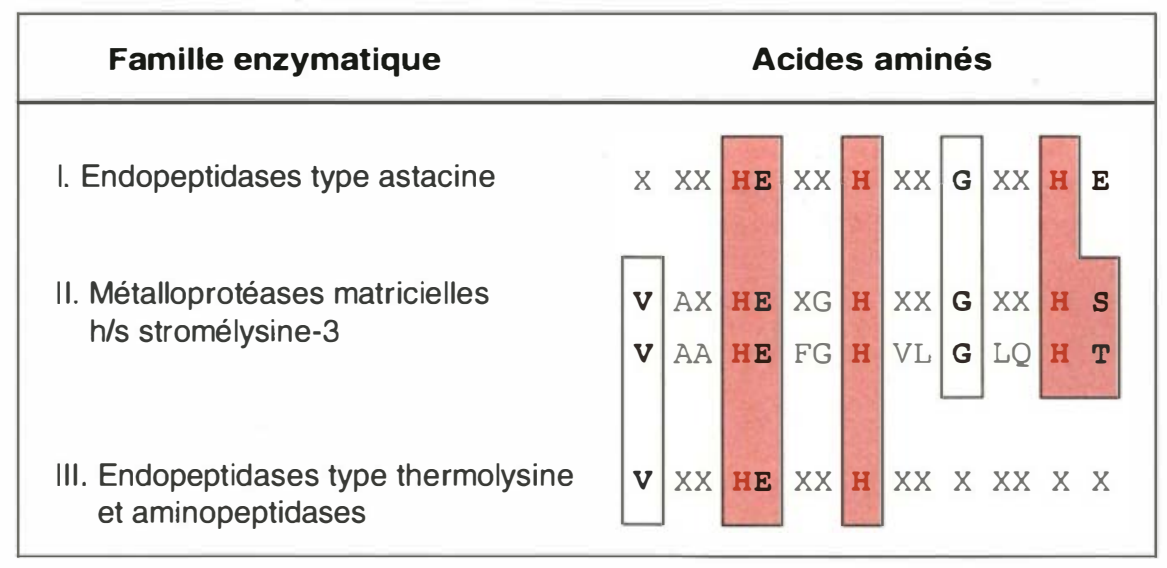

Figure 2. Comparaison des séquences d'acides aminés du domaine de liaison du zinc de certaines métalloprotéases. La liaison de l'atome de zinc du site catalytique des métalloprotéases matricielles à l'apoprotéine fait intervenir trois résidus histidines $(H)$, selon une disposition semblable à celle récemment démontrée pour l'astacine, une métalloprotéase de l'écrevisse [13]. Le site de liaison du zinc de la stromélysine-3 ( $h=$ humaine; $s=$ souris) est analogue à celui des autres métalloprotéases matricielles, sauf en ce qui concerne la sérine (S) adjacente au troisième résidu histidine, qui est remplacée par un thréonine (T). Bien que cette substitution soit conservative, il faut noter que la nature de l'acide aminé faisant suite au troisième résidu histidine est caractéristique de chacune des classes de métalloprotéases se rattachant à la famille de l'astacine [13]. Les métalloprotéases matricielles possèdent un deuxième atome de zinc, dit structural, lié à trois autres résidus histidine qui ne sont pas retrouvés dans les endopeptidases du type astacine [14]. Les métalloprotéases matricielles se distinguent plus nettement des endopeptidases de la famille de la thermolysine et de certaines aminopeptidases, dans lesquelles la liaison du zinc catalytique à l'apoenzyme ne fait intervenir que deux résidus histidines [13]. Les acides aminés sont représentés selon la nomenclature à une lettre: A: Ala; $E$ : Glu; F: Phe; G: Gly; H: His ; L: Leu; $Q: G I n ; S:$ Ser; T:Thr; V: Val; (X désignant les acides aminés non conservés); les acides aminés les plus proches de l'extrémité aminoterminale sont à gauche.

humains et, à cet argument quantitatif et corrélatif, s'ajoute l'observation que l'inhibition de ces enzymes permet de ralentir l'invasion tumorale ou la dissémination métastatique dans un certain nombre de systèmes expérimentaux [8-11].

\section{Le système de la plasmine}

La plasmine est une protéase à sérine capable de cliver la fibrine et la plupart des constituants protéiques de la matrice extracellulaire [8]. La plasmine dérive d'un précurseur inactif, le plasminogène, par action protéolytique d'autres $\mathrm{m} / \mathrm{s} n^{\circ} 5$ vol. 10 , mai 94 pas entièrement connu, mais les observations les plus récentes suggèrent que l'activation du zymogène* implique sa fixation à un récepteur de forte affinité associé à la membrane cytoplasmique de certaines cellules [12]. Finalement, la forme active de l'urokinase est contrôlée par l'expression de plusieurs inhibiteurs protéiques, dont les plus importants sont PAI-1 et PAI-2 (plasminogen activator inhibitor -1 and -2 , $[8])$.

\section{Les métalloprotéases matricielles}

Les métalloprotéases constituent une vaste famille de protéases caractérisées par la présence d'un atome de zinc dans leur site actif. Bien que de nombreuses métalloprotéases soient actives sur la matrice extracellulaire, il est classique de réserver l'appellation métalloprotéase matricielle à un groupe d'enzymes comprenant actuellement neuf membres distincts chez l'homme (figure 1, [10]). Les métalloprotéases matricielles sont caractérisées par la présence d'une cystéine dans leur prodomaine et d'un site de liaison au zinc comparable à celui de l'astacine, une métalloprotéase de l'écrevisse dans laquelle l'atome de zinc est coordonné à trois résidus histidine (figure $2,[13,14]$ ). La cystéine du prodomaine coordonne une quatrième valence de l'atome de zinc, et cette interaction est responsable de la latence des formes proenzymatiques dont l'activation se fait dans le compartiment extracellulaire, par coupure protéolytique entre le domaine catalytique et le prodomaine $[10,15]$. Considérées globalement, les métalloprotéases matricielles sont capables d'agir sur la quasitotalité des constituants protéiques de la matrice extracellulaire, et ces activités sont contrôlées par plusieurs inhibiteurs protéiques dont les plus importants et les mieux caractérisés sont TIMP-1 et TIMP-2 (tissue inhibitor of metalloproteinase -1 and -2 , [15]).

dans la fibranse ex prépondérant L'urokinase est constituée de deux chaînes polypeptidiques reliées par un pont disulfure et produites par clivage protéolytique d'un précurseur monomérique. I.e mécanisme d'activation de la pro-urokinase n'est
* Zymogène: grain de sécrétion intracytoplasmique, protëique, correspondant à des proenzymes. 


\section{RÉFÉRENCES}

12. Blasi F. Urokinase and urokinase receptor: a paracrine/autocrine system regulating cell migration and invasiveness. Biolissays 1993 ; 2: 105-11.

13. Bode W, Gomis-Rüth FX, Huber R, Zwilling R, Stōcker W. Structure of astacin and implications for activation of astacins and zinc-ligation of collagenases. Nalure 1992 ; 358: 164-7.

14. Gooley PR, Johnson BA, Marcy Al, Cuca GC, Salowe SP, Hagmann WK, Esser CK, Springer GP. Secondary structure and zinc ligation of human recombinant shortform stromelysin by multidimensional heteronuclear NMR. Biochemistry 1993; 32 : 13098-108.

15. Docherty AJP, O'Connell J, Crabbe T, Angal S, Murphy G. The matrix metallo proteinases and their natural inhibitors : prospects for treating degenerative tissue diseases. Tibtech 1992; 10: 200-7.

16. He C, Wilhelm SM, Pentland AP, Marmer BL, Grant GA, Eisen AZ, Goldberg GI Tissue cooperation in a proteolytic cascade activating human interstitial collagenase. Proc Nall Acad Sci USA 1989; 86: 2632-6.

17. Ogata Y, Enghild IJ, Nagase H. Matrix metalloproteinase 3 (stromelysin) activates the precursor for the human matrix metalloproteinase 9. J Biol Chem 1992; 267 : 3581-4.

18. Muller D, Wolf C, Abecassis J, Millon R, Engelmann A, Bronner G, Rouyer N, Rio MC, Mehtlin G, et al. Increased stromelysin-3 gene expression is associated with increased local invasiveness in head and neck squamous cell carcinomas. Cancer Res 1993; 53 : 165-9.

19. Wolf C, Rouyer N, Lutz Y, Adida C, Loriot M, Bellocq JP, Chambon P, Basset P. Stromelysin-3 belongs to a subgroup of proteinases expressed in breast carcinoma fibroblastic cells and possibly implicated in tumor progression. Proc Nall Acad Sci USA 1993 ; $90: 1843-7$.

20. Kleiner DE Jr, Stetler-Stevenson WG. Structural biochemistry and activation of matrix metalloproteases. Curr Op Cell Biol

\section{Action synergique des protéases extracellulaires}

Un certain nombre d'observations suggèrent que l'activation des métalloprotéases matricielles pourrait se faire selon une cascade protéolytique impliquant la plasmine [8]. Il faut toutefois remarquer que ce schéma est en partie fondé sur des analyses enzymatiques pratiquées in vitro, dont les résultats ne sont pas forcément transposables à la situation prévalant in vivo. Ainsi, le rôle proposé pour la stromélysine-1 dans l'activation de la procollagénase interstitielle de type I et de la progélatinase B (figure 3, [16, 17]) semble difficilement transposable aux carcinomes humains qui ne sont généralement pas associés à une expression accrue de stromélysine-1 [18, 19], contrai- rement à certaines tumeurs expérimentales chez l'animal [10]. Par ailleurs, l'activation de la progélatinase A in vivo semble être indépendante du système de la plasmine, et impliquer un activateur membranaire spécifique $[15,20]$ distinct du récepteur de forte affinité récemment décrit pour la gélatinase $A$ [21].

L'obtention de souris dans lesquelles l'expression des gènes de l'urokinase et/ou des métalloprotéases matricielles aura été réprimée par recombinaison homologue permettra de tester la validité de ces hypothèses, et en particulier de vérifier s'il existe, ou non, une action synergique entre les deux systèmes protéolytiques in vivo. Une hypothèse alternative est en effet que les deux systèmes sont redondants et représentent un dispositif de sécurité pour les cellules.

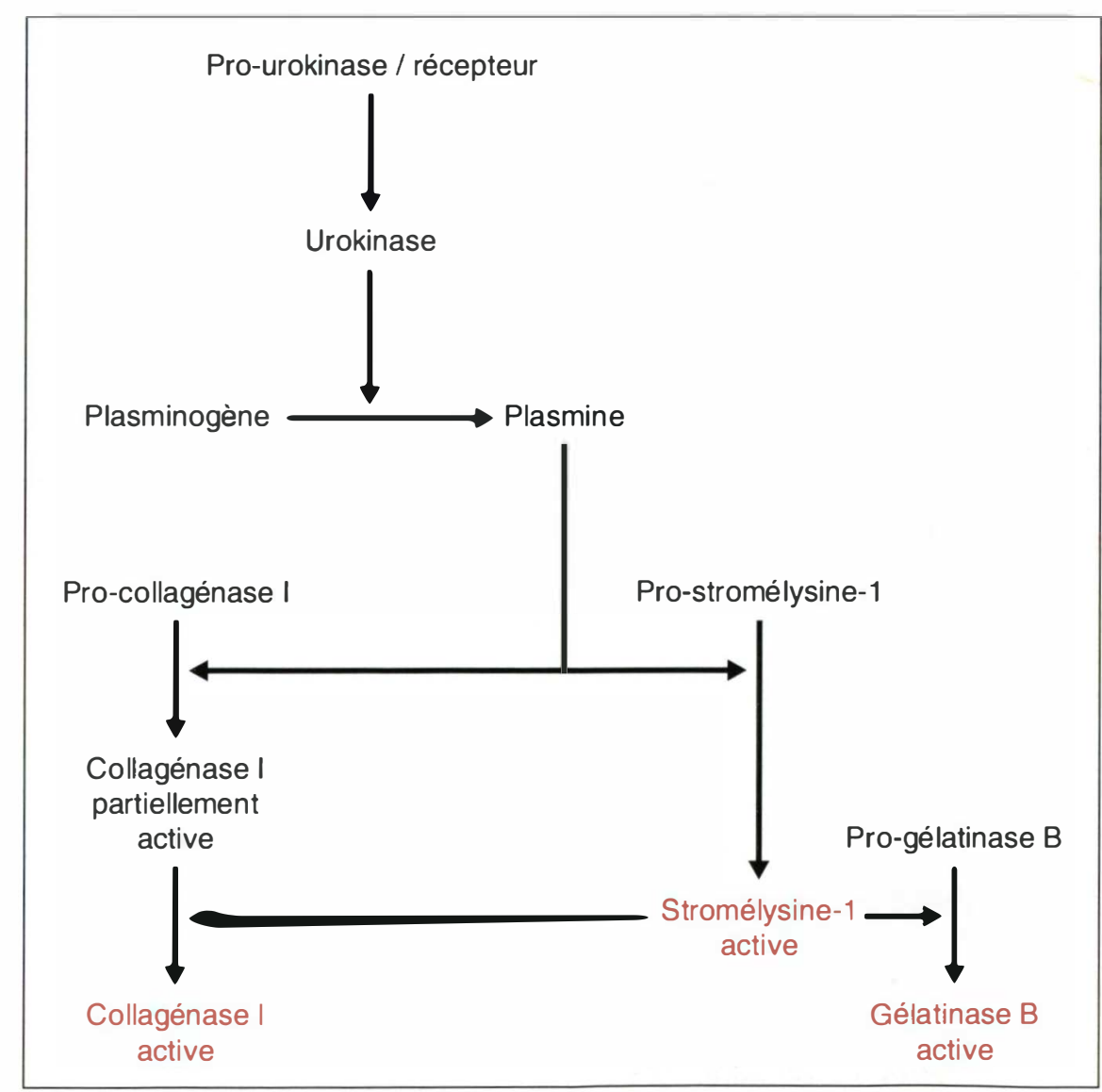

Figure 3. Activation en cascade des protéases extracellulaires. Dans ce schéma hypothétique, la cascade protéolytique est déclenchée par la fixation de la pro-urokinase sur son récepteur membranaire. (D'après [12, 16, 17].) 
Tableau I

EXPRESSION DES GĖNES DE PROTÉASES EXTRACELLULAIRES DANS LES CARCINOMES HUMAINS

\begin{tabular}{|l|c|c|c|}
\hline & Adénocarcinomes & $\begin{array}{c}\text { Carcinomes } \\
\text { épidermoïdes }\end{array}$ & $\begin{array}{c}\text { Carcinomes } \\
\text { basocellulaires }\end{array}$ \\
\hline $\begin{array}{l}\text { Stromélysine-1 } \\
\text { Stromélysine-2 }\end{array}$ & - & - & - \\
Stromélysine-3 & $\mathrm{C}$ & $\mathrm{C}$ & $\overline{\mathrm{F}}$ \\
Matrilysine & $\mathrm{F}$ & $\mathrm{F}$ & $\mathrm{F}$ \\
Collagénase 1 interstitielle & $\mathrm{C}$ & $\mathrm{F}+\mathrm{C}$ & $\mathrm{F}$ \\
Gélatinase A & $\mathrm{F}$ & $\mathrm{F}$ & $\mathrm{I}$ \\
Gélatinase B & $\mathrm{F}$ & $\mathrm{C}$ & $\mathrm{C}$ \\
Urokinase & $\mathrm{F}+\mathrm{C}$ & $\mathrm{C}$ & $\mathrm{C}$ \\
\hline
\end{tabular}

Expression (ARN) par: $F$, cellules fibroblastiques; $C$, cellules cancéreuses; $c$, quelques cellules cancéreuses; I, cellules inflammatoires; -, expression non détectée (d'après [18, 19, 22, 24, 27-31], et les références citées dans ces articles). Deux autres gènes de protéases extracellulaires (collagénase I des neutrophiles et métalloélastase macrophagique, [10]) pourraient être également exprimés par les cellules du stroma des carcinomes humains.

\section{Le paradigme}

\section{de la stromélysine-3}

La stromélysine-3, telle que sa structure peut être déduite de la séquence de son $\mathrm{ADNc}$ [22], est une métalloprotéase matricielle à quatre domaines protéiques, se rattachant de ce point de vue au sous-groupe formé par les autres stromélysines, les collagénases de type I et la métalloélastase macrophagique (figure 1 et [10]). En revanche, la stromélysine-3, qui n'est pas active vis-à-vis de substrats généraux telles la caséine ou la gélatine, se distingue des autres métalloprotéases matricielles par ses propriétés fonctionnelles. Bien que certaines formes enzymatiques tronquées à leur extrémité C-terminale présentent une activité modérée de type stromélysine, il n'est pas certain que ce profil enzymatique soit partagé par la forme mûre complète de l'enzyme [23].

Dans les carcinomes humains, l'ARN et la protéine stromélysine-3 sont exclusivement détectés dans les cellules fibroblastiques du stroma tumoral, au voisinage immédiat des îlots de cellules cancéreuses. Cette observation, faite initialement dans les cancers du sein invasifs (figure 4A $a$ $c,[19,22])$, a été retrouvée dans la plupart des autres types de carcinomes humains (résultats non publiés), et les tumeurs les plus agressives localement sont généralement celles $\mathrm{m} / \mathrm{s} n^{\circ} 5$ vol. 10, mai 94 exprimant les taux les plus élevés de stromélysine-3 [18, 24]. L'expression de la stromélysine-3 peut également s'observer dans certaines tumeurs non invasives, et notamment dans les carcinomes mammaires in situ. Dans ce cas, toutefois, l'expression est inconstante, souvent focale, et moins intense que dans les carcinomes invasifs (figure 4A $d$-f et [19]). Il est également important de noter que l'expression de la stromélysine-3, comme celle des autres métalloprotéases matricielles $[10,15]$, n'est pas spécifique des carcinomes et peut s'observer dans un certain nombre de processus physiologiques, comme l'involution de la glande mammaire [25], la croissance placentaire [22], le développement embryonnaire [22, 25] et la cicatrisation [24].

\section{Autres protéases extracellulaires d'origine stromale}

L'expression de la stromélysine-3 par des cellules de la composante stromale des carcinomes humains fut initialement considérée comme une propriété spécifique de la nouvelle enzyme [22]. Il était en effet admis que les protéases susceptibles d'être impliquées dans la progression tumorale étaient préférentiellement exprimées par les cellules cancéreuses $[5,26]$, et l'expression fibroblas- tique de la collagénase interstitielle de type I dans les carcinomes basocellulaires [27] était généralement perçue comme une exception, voire une curiosité. Ce concept, fondé sur l'étude de lignées cellulaires cancéreuses et sur des analyses immunohistologiques, a été fortement remis en question par les résultats des expériences d'hybridation in situ d'ARNm pratiquées sur coupes histologiques de tumeurs humaines. Ces résultats ont en effet montré que les ARN' de l'urokinase et de la gélatinase A, comme celui de la stromélysine-3, étaient détectés dans les cellules fibroblastiques du stroma tumoral ([19, 28-30] et références citées dans ces articles). Dans les adénocarcinomes mammaires, les gènes de l'urokinase et de la stromélysine-3 sont souvent exprimés par la même population de cellules fibroblastiques (figure $4 B d-i$ ), au voisinage immédiat des îlots de cellules cancéreuses, alors que le gène de la gélatinase A est généralement exprimé par un plus grand nombre de cellules, dont certaines sont situées à distance des cellules cancéreuses (figure $4 B a-c$ ). Ces observations, transposables à la plupart des adénocarcinomes, ne se vérifient que partiellement dans les carcinomes épidermoïdes, puisque dans ces derniers le gène de l'urokinase est exprimé par les cellules cancéreuses ([31] et C.W. résultats non publiés). 


\section{RÉFÉRENCES}

21. Emonard HP, Remacle AG, Noël A, Grimaud JA, Stetler-Stevenson WG, Foidar JM. Tumor cell surface-associated binding site for the $M_{r} 72,000$ type IV collagenase. Cancer Res 1992 ; 52 : 5845-8.

22. Basset P, Bellocq JP, Wolf C, Stoll I Hutin P, Limacher JM, Podhajcer OL, Chenard MP, Rio MC, Chambon P. A novel metalloproteinase gene specifically expressed in stromal cells of breast carcinomas. Nature 1990 ; 348: 699-704.

23. Murphy G, Segain JP, O'Shea M, Cockett $M$, Ionnou $C$, Lef ebvre $O$, Chambon $\mathrm{P}$, Basset $\mathrm{P}$. The $28 \mathrm{kDa}$ N-terminal domain of mouse stromelysin-3 has the general properties of a weak metallopro teinase. J Biol Chem 1993; 268 : 15435-41.

24. Wolf C, Chenard MP, Durand de Grossouvre P, Bellocq JP, Chambon P, Basset $P$. The breast cancer-associated stromelysin-3 gene is expressed in basal cell carcinoma and during cutaneous wound healing. J Invest Dermatol 1992 ; 99 : 870-2.

25. Lef ebvre O, Wolf C, Limacher J, Hutin $\mathrm{P}$, Wendling $\mathrm{C}$, LeMeur $\mathrm{M}$, Basset $\mathrm{P}$, Rio MC. The breast cancer-associated stromelysin-3 gene is expressed during mouse mammary gland apoptosis. J Cell Biol 1992 ; 119 : 997-1102.

26. Monteagudo C, Merino MJ, San-Juan J, Liotta LA, Stetler-Stevenson WG. Immunohistochemical distribution of type IV collagenase in normal, benign, and malignant breast tissue. $A m$ J Pathol 1990; 136 : 585-92.

27. Goslen JB, Bauer EA. Basal cell carcinoma and collagenase. I Dermatol Surg Oncol $1986 ; 12$ : 812-7.

28. Pyke C, Kristensen P, Ralfkiaer E, Grondahl-Hansen J, Eriksen J, Blasi F, Dano K. Urokinase-type plasminogen activator is expressed in stromal cells and its receptor in cancer cells at invasive foci in human colon adenocarcinomas. $\mathrm{Am} J$ Pathol 1991 ; 138 : 1059-67.

29. Polette M, Clavel C, Cockett M, Girod de Bentzmann S, Murphy G, Birembaut P. Detection and localization of mRNAs encoding matrix metalloproteinases and their tissue inhibitor in human breast pathology. Invasion Melastasis 1993; 13: 31-7.

30. Pyke C, Ralfkiaer E, Tryggvason K, Dano K. Messenger RNA for two type IV collagenases is located in stromal cells in human colon cancer. Am J Pathol 1993; 142: 359-65.

Des analyses comparables ont été effectuées pour les autres gènes de métalloprotéases matricielles. De ces études, il ressort que l'expression des protéases extracellulaires au cours de la progression des carcinomes semble obéir à un schéma plus complexe que celui initialement proposé, et dans lequel la majeure partie de l'activité protéolytique proviendrait du stroma tumoral (Tableau I). Seuls les gènes de la matrilysine et de la stromélysine-2 sont spécifiquement exprimés par les cellules cancéreuses des carcinomes, indépendamment du type histologique considéré. Toutefois, fautil encore noter que l'expression du gène de la matrilysine peut également s'observer dans les cellules épithéliales non cancéreuses et que celle du gène de la stromélysine-2 est peu fréquente dans les adénocarcinomes [19].

\section{Rôle des protéases d'origine stromale}

L'identification du stroma comme site principal de production des protéases extracellulaires dans les carcinomes ne modifie pas, a priori, l'hypothèse selon laquelle l'action première de ces enzymes au cours de la progression tumorale serait de rompre la barrière représentée par la matrice extracellulaire, au profit de l'expansion des cellules cancéreuses. Ces dernières pourraient contrôler l'expression stromale des protéases par l'intermédiaire de facteurs diffusibles [22, 27], dont l'action pourrait être en partie relayée par le proto-oncogène c-etsl [32]. L'hypothèse est en bon accord avec les observations montrant que les cellules stromales qui expriment le gène de la stromélysine- 3 , et celui de l'urokinase dans les adénocarcinomes, sont situées au voisinage immédiat des îlots de cellules cancéreuses. Il est toutefois important de noter que l'expression des gènes stromélysine-3 et urokinase s'observe également dans certains carcinomes non invasifs (figures $4 A d-g, 4 B g-i$ et [19]), et que l'expression de certaines protéases stromales peut s'observer dans des cellules situées à distance des îlots de cellules cancéreuses [19, 24, 27, 30]. S'il est classique, et a priori logique, d'associer protéolyse extracellulaire et destruction tissulaire, l'analyse des carcinomes

Figure 4. Expression stromale des gènes de la stromélysine-3, de la gélatinase $A$ et de l'urokinase dans les cancers du sein. A. Expression du gène de la stromélysine-3. Photomicrographies de coupes histologiques de carcinomes invasifs a-c, et de carcinomes in situ $d-f$; analyse par hybridation in situ de l'ARN codant pour la stromélysine-3 $(a, b, d, e)$ et analyse immunohistologique de la protéine stromélysine-3 (c, f). ARN et protéine sont spécifiquement détectés dans les cellules fibroblastiques du stroma tumoral au voisinage immédiat des illots de cellules cancéreuses lb et e sont des photomicrographies sur fond noir, correspondant respectivement aux images présentées en a et d). B. Expression comparative des ARN stromélysine-3, gélatinase $A$ et urokinase. Photomicrographies de coupes histologiques de carcinomes. invasifs (a-f), et de carcinomes in situ $(g-i) ; b, c, e, f, h, i$ sont des photographies sur fond noir; a-c, d-f et $g$-i, correspondent à trois séries distinctes de coupes sériées: I'ARN de la gélatinase $A(c)$ est détecté dans un grand nombre de cellules stromales dont certaines sont situées à distance de la zone de carcinome invasif (IC), contrairement à I'ARN stromélysine-3 (b) dont l'expression est restreinte au stroma de la zone tumorale invasive; les profils d'expression des ARN de l'urokinase (f, i) et de la stromélysine-3 $(e, h)$ sont, en revanche, très comparables, tant dans les carcinomes invasifs ( $d-f)$ que dans les carcinomes in situ $(g-i)$. (Le panneau $B$ est une reproduction avec autorisation d'une illustration provenant de [19], à laquelle le lecteur est également renvoyé pour les informations techniques.)

Abréviations: $A$, adventice d'un vaisseau sanguin $(V) ; C$, cellules cancéreuses invasives; IC, zone de cancer invasif, associant cellules cancéreuses et cellules stromales; $L$, lobule normal; $S$, stroma tumoral; *, zone de carcinome in situ. 

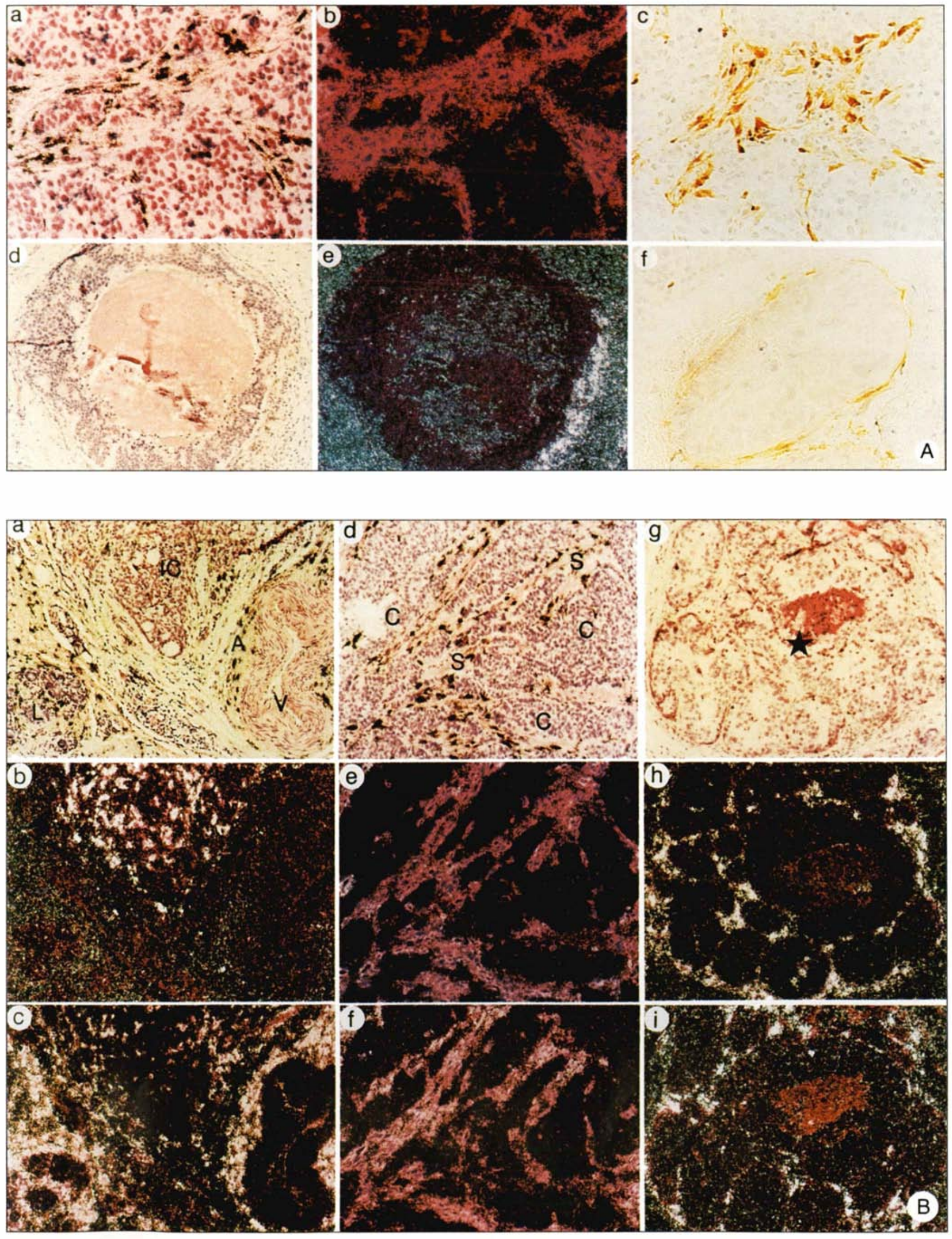


\section{RÉFÉRENCES}

31. Sappino AP, Belin D, Huarte J, Hirschel-Scholz S, Saurat JH, Vassalli JD Differential protease expression by cutaneous squamous and basal cell carcinomas. J Clin Invest 1991 ; 88 : 1073-9.

32. Wernert $N$, Raes $M B$, Lassalle $P$, Dehouck MP, Gosselin B, Vandenbunder $\mathrm{B}$, Stéhelin D. cetsl proto-oncogene is a transcription factor expressed in endothelial cells during tumor vascularization and other forms of angiogenesis in humans. Am J Pathol 1992; 140: 119-27.

33. Pepper MS, Sappino AP, Stōcklin R, Montesano R, Orci L, Vassalli JD. Upregulation of urokinase receptor expression on migrating endothelial cells. J Cell Biol 1993 . 122: $673-84$.

34. Sullivan LM, Quigley JP. An anticataly tic monoclonal antibody to avian plasminogen activator: its eff ect on behavior of RSV-transformed chick fibroblasts. Cell $1986 ; 45: 905-15$.

35. Hecht PM, Anderson KV. Extracellular proteases and embryonic pattern formation. Trends Cell Biol $1992 ; 2$ : 197-202

36. Naldini L, Tamagnone L, Vigna E, Sachs M, Hartmann G, Birchmeier W, Daikuhara Y, Tsuboushi H, Blasi F, Comoglio PM. Extracellular proteolytic cleavage by urokinase is required for activation of hepatocyte growth factor scatter factor $E M B O$ J 1992 ; 11 : 4825-33.

37. Odekon LE, Sato Y, Rifkin DB. Urokinase-type plasminogen activator mediates basic fibroblast growth factorinduced bovine endothelial cell migration independent of its proteolytic activity. J Cell Physiol 1992; 150: 258-63.

38. Bard J. The cellular and molecular processes of developmental anatomy. Mor phogenesis. Cambridge: University Press, 1990: 120-80.

39. Brown PD. Matrix metalloproteinase inhibitors : a new class of anticancer agent.

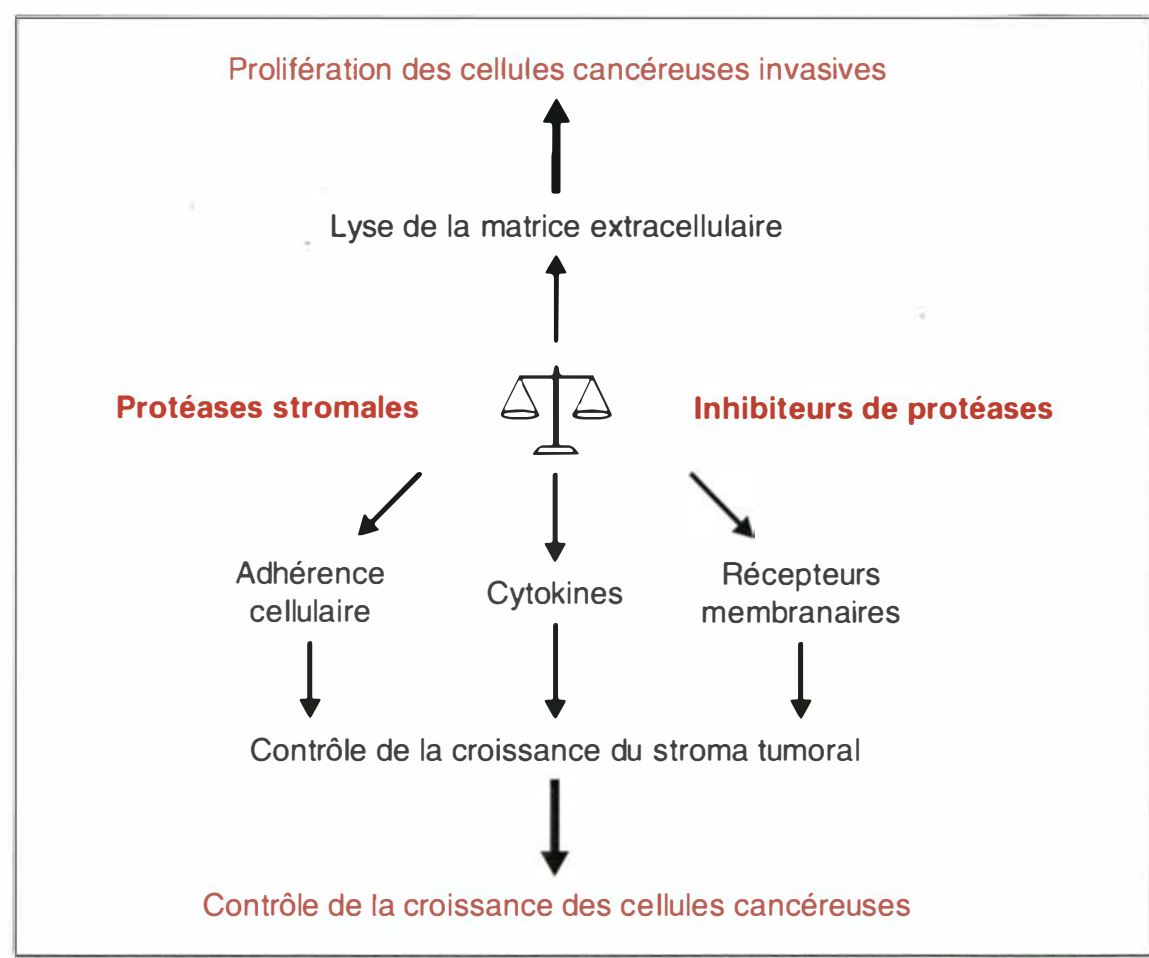

Figure 5. Mécanismes potentiels de l'action des protéases extracelIulaires d'origine stromale au cours de la progression des carcinomes. Dans ce modèle, la balance protéolytique, fonction du rapport existant entre les formes actives de protéases extracellulaires et leurs inhibiteurs, apparaît comme le chef d'orchestre de la croissance tumorale. Les protéases extracellulaires étant essentiellement produites par les cellules stromales des carcinomes, l'action première de ces protéases pourrait concerner la croissance de la composante stromale des tumeurs, laquelle précède généralement la phase d'invasion tumorale proprement dite. En participant à la mise en place et au maintien du stroma tumoral, les protéases d'origine stromale contribueraient indirectement à la progression tumorale. Une action plus directe, s'exerçant sur la matrice extracellulaire au contact des cellules cancéreuses, pourrait également contribuer à l'expansion des cellules cancéreuses hors du compartiment épithélial.

humains montre que les protéases d'origine stromale sont en fait exprimées dans un compartiment tissulaire où les aspects trophiques sont plus apparents que les phénomènes destructifs, y compris dans les tumeurs invasives. Comme nous l'avons souligné précédemment, le stroma des carcinomes fait partie intégrante de la néoformation tumorale, dont il représente la composante non épithéliale, et il ne doit pas être assimilé au tissu conjonctif situé en périphérie de la tumeur. Prises dans leur ensemble, ces observations suggèrent que les protéases d'origine stromale pourraient, indé- pendamment ou secondairement à leur action sur la matrice extracellulaire, contribuer au développement du compartiment tumoral dans lequel elles sont exprimées. Le concept selon lequel les protéases d'origine stromale pourraient avoir une action trophique sur le stroma tumoral est en fait implicitement admis puisque ce dernier est le siège de la néovascularisation tumorale, dont on pense qu'elle fait intervenir l'urokinase, son récepteur et l'inhibiteur du plasminogène de protéases d'origine stromale pourrait toutefois ne pas se limiter au type 1 [33]. L'action trophique des 
système vasculaire, et concerner également les cellules fibroblastiques qui représentent souvent un important contingent cellulaire de la composante stromale des carcinomes. En diminuant transitoirement l'adhérence des fibroblastes à la matrice extracellulaire, les protéases extracellulaires pourraient favoriser l'accumulation de ces cellules dans le stroma tumoral, en stimulant à la fois leur migration et leur multiplication [10, 34]. Les protéases extracellulaires pourraient également agir par l'intermédiaire de l'activation de cytokines associées à la matrice extracellulaire $[8,35,36]$, et même avoir une activité intrinsèque de facteur de croissance par l'intermédiaire de l'activation de récepteurs membranaires. Une telle activité a été démontrée pour la thrombine [35] et pourrait également concerner l'urokinase [37]. A cet égard, il est important de souligner qu'un certain nombre de facteurs, initialement identifiés par leur action trophique au cours du développement embryonnaire ou de la réparation tissulaire, se sont révélés par la suite être des protéases [35]. Un exemple classique est celui de la protéine morphogénétique de l'os (BMP-1) qui appartient à la famille de l'astacine, une protéase à laquelle se rattachent, comme nous l'avons vu précédemment, la stromélysine- 3 et les autres métalloprotéases matricielles.

\section{Conclusion}

\section{et perspectives}

L'analyse directe des carcinomes humains, grâce aux outils puissants et précis de la génétique moléculaire, a permis de conforter l'hypothèse selon laquelle la composante stromale de ces tumeurs est impliquée, au même titre que les cellules cancéreuses, dans la croissance tumorale. Le stroma est le siège de la néovascularisation tumorale, mais il est vraisemblable qu'il ne s'agit là que d'un des aspects par lequel il contribue à la progression des carcinomes. L'induction d'un stroma distinct du tissu conjonctif normal est en effet un événement précoce de la progression tumorale, qui rappelle les condensations stromales du développement embryonnaire [38] ou les modifications tissulaires accompagnant la cicatrisation [4]. L'identification du stroma des carcinomes comme principal site de synthèse des protéases extracellulaires suggère que l'action de ces enzymes pourrait ne pas se limiter à la lyse de la matrice extracellulaire au profit de l'expansion des cellules cancéreuses, mais impliquer une fonction plus complexe de régulation de la croissance tumorale, dont une partie s'exercerait sur les cellules du stroma elles-mêmes (figure 5). Une meilleure caractérisation des mécanismes moléculaires intervenant dans cette action pourrait conduire au développement de moyens thérapeutiques complémentaires de ceux dont on dispose à l'heure actuelle, et qui sont généralement conçus pour la destruction des seules cellules cancéreuses. A cet égard, l'utilisation d'inhibiteurs des métalloprotéases matricielles [11, 15, 39] pourrait offrir, à moyen terme, de nouvelles perspectives thérapeutiques

\section{Summary}

Stromal proteinases and tumor progression

Recent observations, based on mRNA in situ hybridization studies, have shown that the main source of extracellular proteolytic activity in most human carcinomas comes from stromal cells of tumors, and not from cancer cells as was believed. These observations suggest that the role of extracellular proteinases during tumor progression may be more complex than previously proposed, and strengthen the concept that future approaches to cancer treatment should be clirected at molecular mechanisms specifically interfering with the tumor stroma.

\section{TIRÉS A PART}

P. Basset. 